\title{
Non-radial pulsations in the CoRoT Be Star 102761769
}

\author{
Eduardo Janot Pacheco ${ }^{1}$, Laerte B.P. de Andrade ${ }^{1}$, Marcelo Emilio ${ }^{2}$, \\ Juan Carlos Suárez ${ }^{3}$ and Andressa Jendreieck ${ }^{1}$ \\ ${ }^{1}$ Instituto de Astronomia, Geofísica e Ciências Atmosféricas, Universidade de São Paulo, \\ Rua do Matão 1226, 05508-090, São Paulo/SP, Brazil \\ ${ }^{2}$ Observatorio Astronomico/DEGEO, Universidade Estadual de Ponta Grossa, \\ Av. Carlos Cavalcanti, 4748 Ponta Grossa, / PR, Brazil - 84030-900 \\ ${ }^{3}$ Instituto de Astrofísica de Andalucía (CSIC), Glorieta de la \\ Astronomía, E-18008 Granada, Spain
}

\begin{abstract}
We investigate non-radial pulsations of the CoRoT IR1 Be Star 102761769, with a projected stellar rotation estimated to be $120 \pm 15 \mathrm{~km} / \mathrm{s}$. If the star is a typical galactic Be star it rotates near the critical velocity. We propose an alternative scenario, where the star could be seen nearly equator-on rotating at a relatively moderate velocity say, $\approx 120 \mathrm{~km} / \mathrm{s}$ and therefore the nonradial oscillations could be modeled. In order to identify the pulsation modes of the observed frequencies, we computed a set of models representative of CoRoT 102761769 by means of the adiabatic pulsation package FILOU. Results indicate that the two frequencies are compatible with a high-g mode as predicted by pulsation models of Be stars.
\end{abstract}

Keywords. stars: oscillations (including pulsations), stars: rotation, stars: emission-line, Be

\section{Photometric and spectroscopic observations}

In this work we investigate the variability of the CoRoT IR1 Be Star 102761769 observed in the exoplanet field during 54.6 days. Time series analysis of the light curve was made using both Cleanest and Singular Spectrum Analysis algorithms. We found two close frequencies related to the star at $f_{1}=2.465 \mathrm{c} / \mathrm{d}(28.5 \mu \mathrm{Hz})$ and $f_{2}=2.441 \mathrm{c} / \mathrm{d}$ $(28.2 \mu \mathrm{Hz})$. The precision to which those frequencies were found is $0.018 \mathrm{c} / \mathrm{d}(0.2 \mu \mathrm{Hz})$. One low-resolution spectrum of the star was obtained at Observatorio del Roque de los Muchachos (La Palma). The projected stellar rotation is estimated to be $120 \mathrm{~km} / \mathrm{s} \pm$ $15 \mathrm{~km} / \mathrm{s}$ from the Fourier transform of spectral lines. If CoRoT 102761769 is a typical galactic Be star it rotates near the critical velocity. The critical rotation frequency of a typical B5-6 star is about $3.5 \mathrm{c} / \mathrm{d}(40.5 \mu \mathrm{Hz})$, suggesting that the above frequencies are really due to stellar pulsations rather than linked to the star's rotation.

\section{Representative models}

In order to identify the pulsation modes of the observed frequencies in the star, we computed a set of asteroseismic models representative of CoRoT 102761769 by varying its main physical parameters (Emilio et al. 2010): its mass $\left(\mathrm{M}=3.8 \pm 0.2 M_{\odot}\right)$ and equatorial rotation velocity $\left(v_{r o t, s}=90\right.$ or $120 \mathrm{~km} / \mathrm{s}$ ), assuming a solar abundance of elements (Figure 1). The structure models were computed using the evolutionary code CESAM (Morel, 1997), assuming, as would be plausible for such stars, an uniform rotation for the core and differential rotation for the envelope. For this latter, local conservation of the angular momentum during the evolution was assumed (Suárez et al. 2009), resulting in a radial differential rotation profile of the type $\Omega(r)=\Omega_{s}\left[1-\eta_{0}(r)\right]$. 


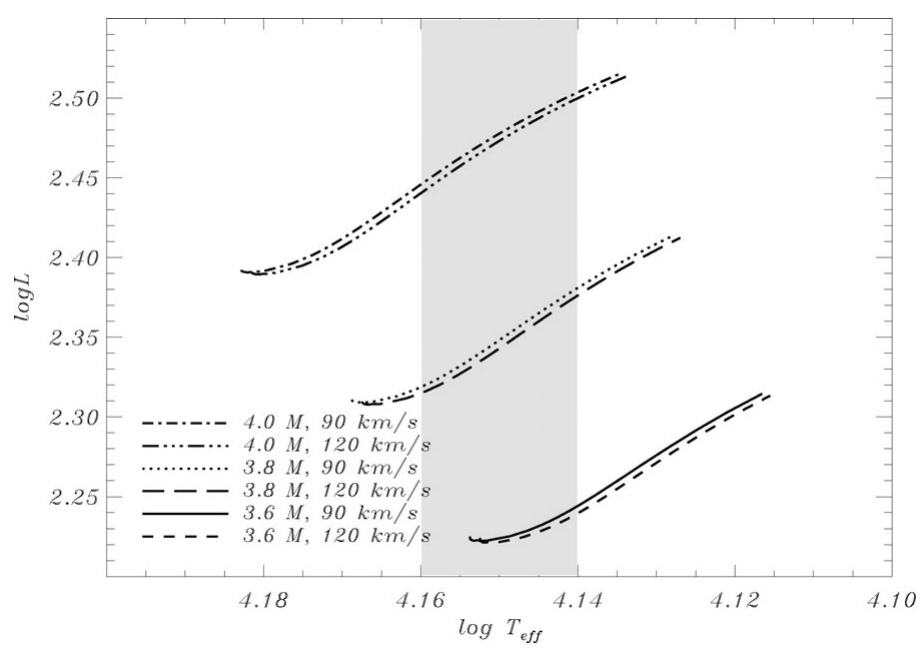

Figure 1. HR diagram showing the tracks of evolutionary paths calculated for CoRoT 102761769 , for three different masses $\left(3.6,3.8\right.$ and $\left.4.0 M_{\odot}\right)$ and two different surface equatorial velocities (90 and $120 \mathrm{~km} / \mathrm{s}$ ). The shaded area corresponds to the uncertainty in effective temperature.

\section{Splittings and asymmetries for low $l$ multiplets}

Oscillation frequencies were then derived using the adiabatic pulsation package FILOU (Tran Minh \& Léon 1995; Suárez \& Goupil 2008) presuming the effects of rotation up to second order, and assuming mode coupling as would be expected of a star with high rotational velocity. The code compensates for the equilibrium models without non-spherical components by means of a linear perturbation analysis. A rotation of $120 \mathrm{~km} / \mathrm{s}$ for an intermediate-mass star can be considered within the limit of validity of the perturbation theory (Suárez et al. 2006). Caution must be taken in any case until similar computations with a non-perturbative oscillation theory can be done for realistic stellar models. The resulting frequency spectra were then scanned for those modes that better approach the observed $f_{1}$ and $f_{2}$, inside an effective temperature uncertainty derived from Huang \& Gies $(2006)$ as $\log \left(T_{e f f}\right)=4.16 \pm 0.01$ for a B5-6V star. The central value considered for the effective temperature was that of a 3.8 solar mass star in the middle of the main sequence. The results indicate that for all the models considered the frequencies can only be described by a high-g mode, $g_{5}(l=1, m=0)$.

\section{Acknowledgements}

The authors would like to acknowledge the financial support of IAG-USP, CNPq and FAPESP.

\section{References}

Emilio, M., Andrade, L., Janot-Pacheco, E., \& Baglin, A. et al. 2010, A\&A, 522A, 43

Huang, W. \& Gies, D. R. 2006, ApJ, 648, 591

Morel, P. 1997, A\& AS, 124, 597

Suárez, J. C., Goupil, M. J., \& Morel, P. 2006, A\&̛A, 449, 673

Suárez, J. C. \& Goupil, M. J. 2008, Ap\&SSS, 316, 155

Suárez, J. C., Moya, A., Amado, P. J., \& Martín-Ruiz, S. et al. 2009, ApJ, 690, 1401

Tran Minh, F., \& Léon, L. 1995, in: I. W. Roxburgh \& J.-L. Masnou (eds.) Physical Processes in Astrophysics, p. 219 